\title{
PERFORMANCE EVALUATION OF DIFFERENT MODULATION SCHEMES FOR ULTRA WIDE BAND SYSTEMS
}

\author{
Tasnuva Ali* — Poppy Siddiqua* — Mohammad A. Matin ${ }^{* *}$
}

\begin{abstract}
Ultra-wideband (UWB) signals with a large bandwidth has some advantages like multipath immunity, low transmission power, good resolution for ranging and detecting geo locations, as well as it can resist to narrow-band interference. These signals are used for transmission in short distance with high throughput. In this paper, we have analyzed different modulation schemes for performance comparison in terms of BER with UWB Gaussian second derivative monopulse and wavelet-based monopulse. The simulation results demonstrate that wavelet-based monopulse provides better performance in comparison to the other two monopulses.

K e y w or d s: UWB, Gaussian monopulse, wavelet-based monopulse, BER, $E_{b} / N_{o}$, PPM, BPSK
\end{abstract}

\section{INTRODUCTION}

Ultra wideband (UWB) is a wireless technology which is capable of transmitting large amounts of digital data over a wide spectrum of frequency band with very low power for a short distance. As it uses extremely wide transmission bandwidth, the UWB signal has the potential for improving the ability to measure location and range accurately, high multiple-access capability, extremely high data rate at short ranges and easier material penetrations. For its extremely low duty cycles, it is immune from multipath effects and fading problems, whereas existing wireless systems suffer those problems [2]. In 2002, the US Federal communications Commission (FCC) approved unlicensed use of the spectral band of UWB from 3.1 to $10.6 \mathrm{GHz}$ with a transmitted power spectral density (PSD) less than $-41.0 \mathrm{dBm} / \mathrm{MHz}$ for indoor wireless communication. Due to the low power density, the distance of UWB communication system is limited to a few meters to tens of meters. Moreover, the effective isotropic radiated power (EIRP) for UWB communication must comply with the regulation defined by Federal Communications Commission (FCC) to coexist with many existing wireless communication systems [11]. It also requires the optimal receiving characteristics, which is to say high fidelity at the receiver side [12].

The main candidates for UWB modulation scheme can be classified into two basic categories; they are time-based modulation and shape-based modulation [3]. Pulse position modulation (PPM) is an example of time-based modulation whereas Binary Phase Shift Keying (BPSK), On-Off Keying (OOK) and pulse Amplitude Modulation (PAM) are the examples of shape-based modulation technique.

Here, we considered different popular modulation schemes such as Binary Phase Shift Keying (BPSK), Pulse Position Modulation (PPM) and On-Off Keying
(OOK). In this paper, Pulse position modulation (PPM) has been considered as a suitable M-ary modulation scheme for its fine time resolution [10]. With PPM, the data modulates the position of the transmitted pulse within an assigned window in time [9]. Here, we estimate the performance of UWB communication system through simulation for M-ary PPM in the presence of additive white Gussian noise (AWGN) channel.

For performance analysis, two types of waveforms; namely, wavelet based monopulse and the 2nd derivative Gaussian pulse are considered in this paper. The BER analysis of the UWB M-ary PPM in additive white Gaussian noise channel for the wavelet based monopulse shows that the lower order modulation scheme gives better result compared to the higher order modulations.

\section{UWB PULSE SHAPES AND MODULATION}

The most widely studied and implemented pulse shapes for UWB communication are Gaussian first derivative, Gaussian second derivative, Wavelet-based monocycle and Manchester monocycle. The choice of pulse shape is a most important consideration in the design of UWB communication systems. The fractional bandwidth of a pulse is defined as the ratio of the signal bandwidth (BW) measured at a fixed power level relative to the peak level at its center frequency [18].

In this paper, Gaussian second derivative and wavelet based monocycle pulses are considered as UWB signal and the transmission of these signal affects the performance of different modulation schemes in the presence of AWGN and Rayleigh fading channel. Gaussian second derivative pulse is considered because its high fractional bandwidth will allow the UWB system to experience much less fading in multipath environment. Again, wavelet based monopulse has the advantage of reduced

\footnotetext{
* Department of ETE, Daffodil International University, Dhaka, Bangladesh ${ }^{* *}$ Department of EEE, Institut Teknologi Brunei, Brunei Darussalam, tasnuva@daffodilvarsity.edu.bd, poppy@daffodilvarsity.edu.bd, matin.mnt@gmail.com
} 


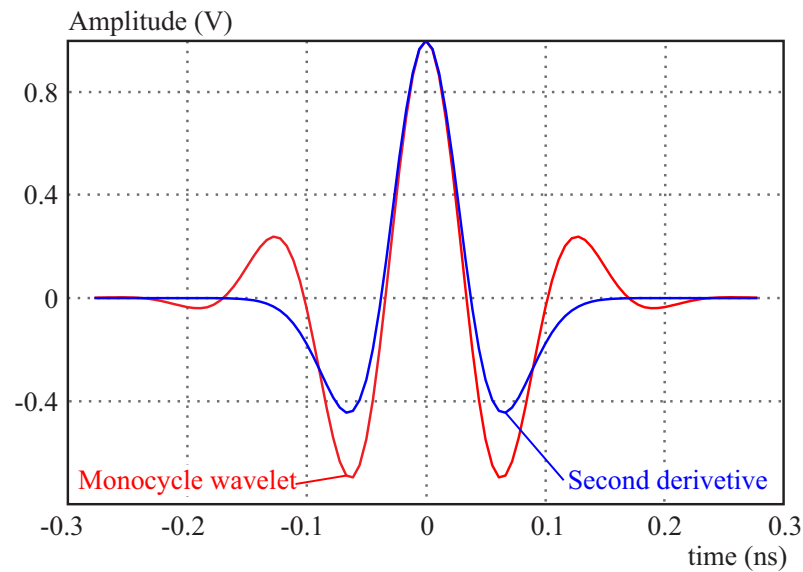

Fig. 1. Pulse shapes in time domain

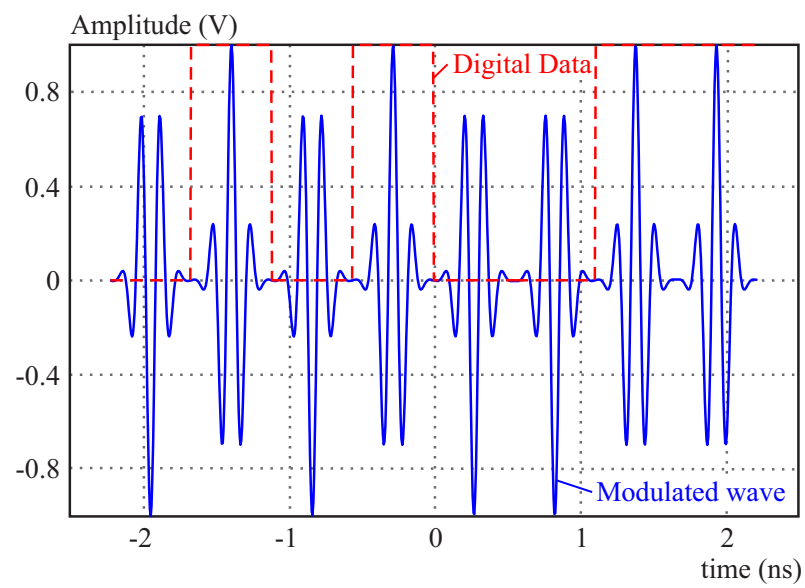

Fig. 2. Pulse train for BPSK modulation using wavelet-base monopulse

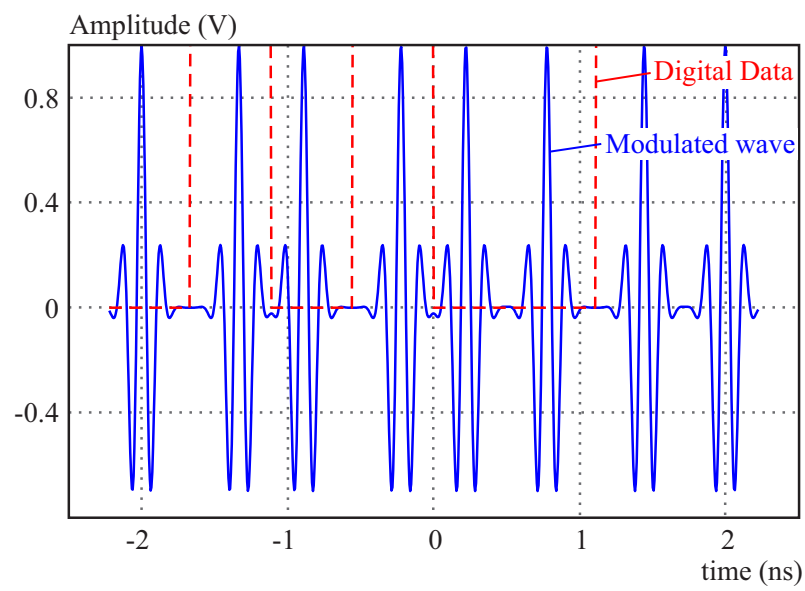

Fig. 3. Pulse train for BPPM modulation using wavelet-base monopulse

interference, which then results in an improved BER performance. These pulse shapes enable the use of a RAKE receiver to receive a stronger signal.

The wavelet based UWB signal can be defined as [1].

$$
w(t)=A_{p} \exp \left(-b\left(\frac{t-\tau}{a}\right)^{2}\right) \times \cos \left(k \pi\left(\frac{t-\tau}{a}\right)\right) .
$$

For large bandwidth, the parameters of $\left(a, f_{0}\right)$ can be chosen as: $f_{0}=0.57, a=7.7444 \times 10^{-11}$. The value of $b=0.5$ is selected in between 0 to $1 \mathrm{~ns}$ to meet the FCC emission mask. We also use 2nd order Gaussian pulse because it is the most currently adopted pulse that meet the appropriate UWB operation which is usually generated by the equation

$$
w(t)=2 \frac{A}{T_{a u}} \sqrt{e}\left(t-T_{c}\right) e^{-2\left[\frac{t-T_{c}}{T_{a u}}\right]^{2}} .
$$

Here, $w(t)$ is a Gaussian second derivative pulse where pulse duration or width is much smaller than pulse repetition period, so it can produce low duty cycle operation. According to Fig. 1, the Gaussian second derivative has two zero crossing points and the relative bandwidth decreases with the increasing of the center frequency [2].

\section{UWB MODULATION SCHEMES}

In general UWB signal is designed to smooth the spectrum of the signal and protect the system from radio interference to the existing narrowband and wideband signals. The wavelet-based monopulse is used to generate pulse train which is modulated using BPSK, PPM and OOK. M-ary PPM modulation scheme needs a very fine time resolution.

\subsection{BPSK Modulation Scheme}

BPSK is the modulation technique where two different phase values indicate two different data symbols. This modulation scheme can be expressed as follows

$$
S^{(k)}(t)=\sum_{j} w\left(t-j T_{f}-\varphi\right),
$$

where, $\varphi$ is the phase which has the values of 0 and 180 degrees for data symbol 1 and 0 respectively. For a given data sequence like $\left\{\begin{array}{llllllll}0 & 1 & 0 & 1 & 0 & 0 & 1 & 1\end{array}\right\}$ we can obtain the wavelet-based pulse train for BPSK as in Fig. 2.

\subsection{PPM Modulation Scheme}

BPPM is the modulation scheme where an additional time shift is used to indicate the change of data symbol. The PSD of PPM can be smoothed by using PN sequence, and the parameters may be chosen to move discrete spectral lines so as to minimize interference into other communication signals [18]. These PN sequence can be used to serve many users simultaneously which provides multiple access (MA) facilities. PPM also offers UWB multiple access communications using AWGN channel with combined transmission capacity of several hundreds of Mbps at BER rates in the range from 10-4 to 10-7 using moderate complexity receivers.

BPPM can be expressed as follows

$$
S^{(k)}(t)=\sum_{j} w\left(t-j T_{f}-c_{j}^{(k)} T_{c}-\delta d^{(k)}\left[j / N_{s}\right]\right)
$$




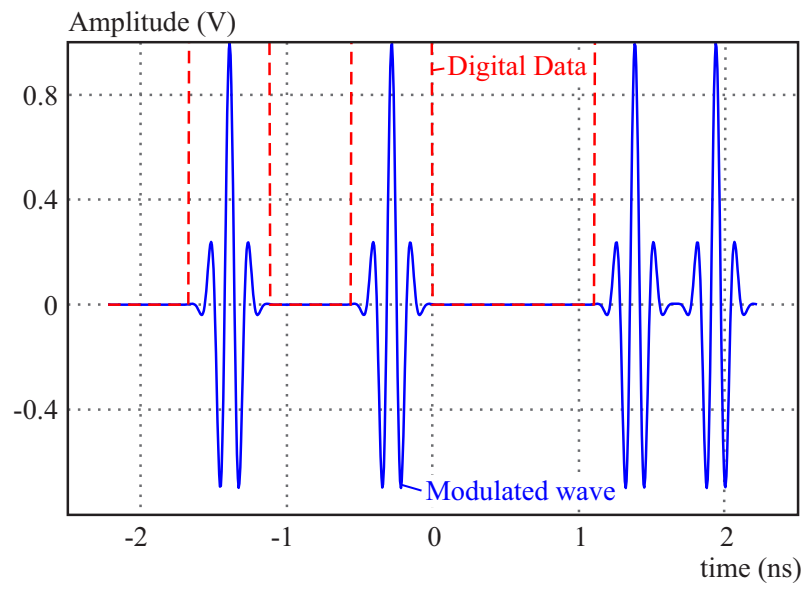

Fig. 4. Pulse train for BPPM modulation using wavelet-based monopulse

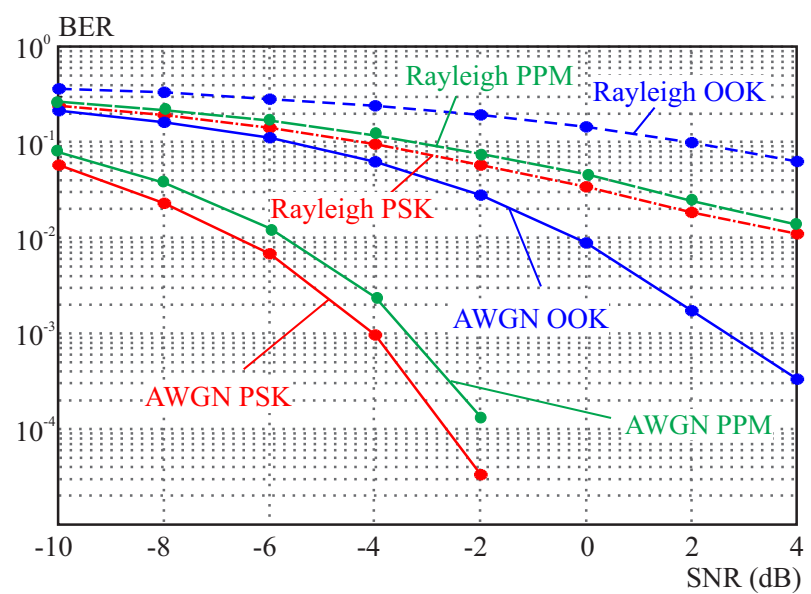

Fig. 6. BER performances in AWGN and Rayleigh channel for three modulation schemes for wavelet based monopulse

where, $C_{j}=0$, since no chip sequence is used and $\delta$ is the modulation factor which indicates 1 for additional time shift and 0 for no time shift. For a given data sequence like $\left\{\begin{array}{llllllll}0 & 1 & 0 & 1 & 0 & 0 & 1 & 1\end{array}\right\}$ we can obtain the wavelet-based pulse train for BPPM as in Fig. 3 .

\subsection{OOK Modulation Scheme}

OOK is the modulation technique where the data symbol ' 1 ' represents the presence of a pulse and ' 0 ' data symbol indicates no pulse. On-off keying modulation can be expressed as follows

$$
S^{(k)}(t)=\sum_{j} d^{(k)} w\left(t-j T_{f}\right) .
$$

For a given data sequence like $\left\{\begin{array}{llllllll}0 & 1 & 0 & 1 & 0 & 0 & 1 & 1\end{array}\right\}$ we can obtain the wavelet-based pulse train for OOK as in Fig. 4.

Our present work focuses on the BER performances of three basic pulse shapes in PPM scheme and wavelet based monopulse turned out to be the best pulse-shape which gives the best BER result. Thus we can say that wavelet-based monopulse is the best UWB pulse shape which can be used for UWB transmission using PPM scheme.

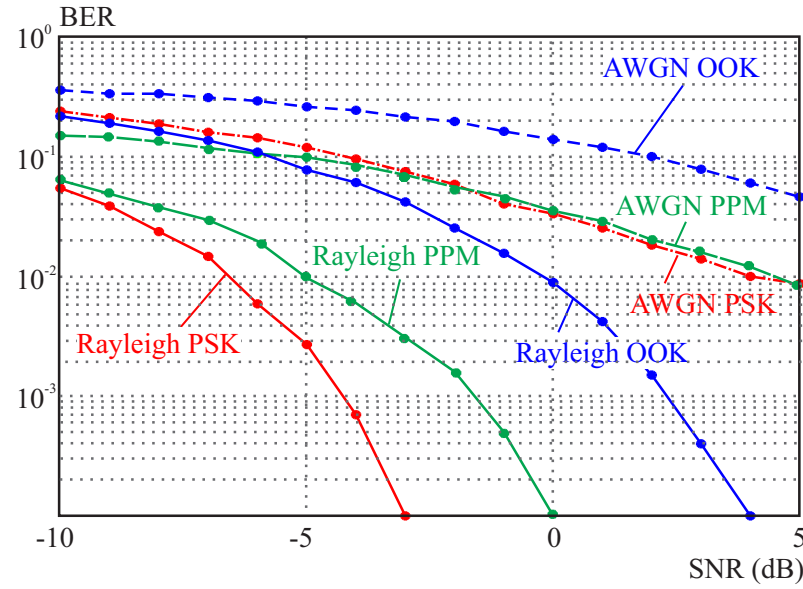

Fig. 5. BER performances for AWGN and Rayleigh channel

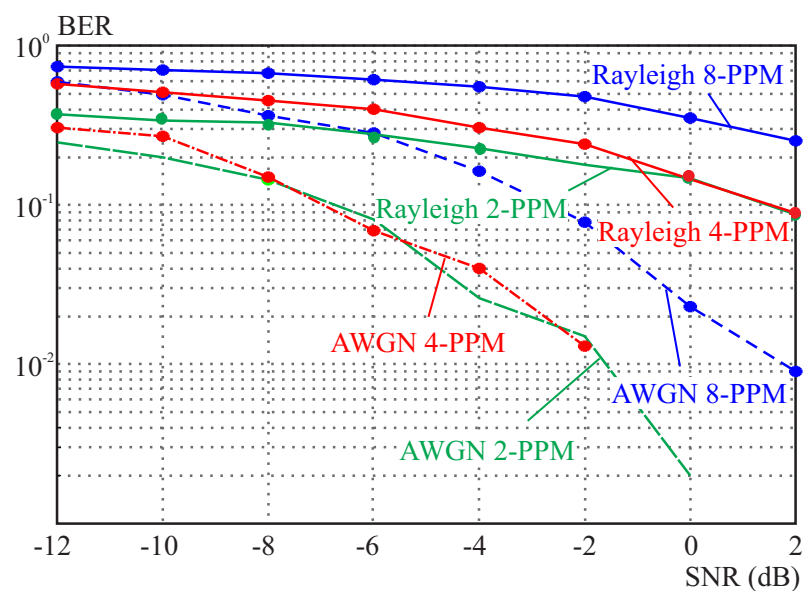

Fig. 7. BER performances for M-ary PPM modulation scheme for wavelet based monopulse

\section{SIMULATION RESULT}

In this paper, three different modulation schemes for two different UWB pulses- Gaussian second derivative and wavelet based monocycle pulse are considered for performance evaluation. The performance is compared in the presence of AWGN and Rayleigh fading channel.

Figure 5 shows that the BER performance of BPSK modulation scheme which gives better performance in AWGN channel as compared to PPM and OOK modulation techniques. That means, for equal energy per bit, BPSK has greater inter-symbol distance than BPPM. Thus BPPM requires more energy to achieve the same bit error rate than BPSK and PPM offers advantages over OOK for Gaussian second derivative pulse shape. Also AWGN channel gives better performance than Rayleigh fading channel for three modulation schemes.

By considering wavelet based monocycle pulse, the BER (bit error rate) performance of the three modulation techniques are compared in the presence of the AWGN (additive white Gaussian noise) and Rayleigh fading channel. 


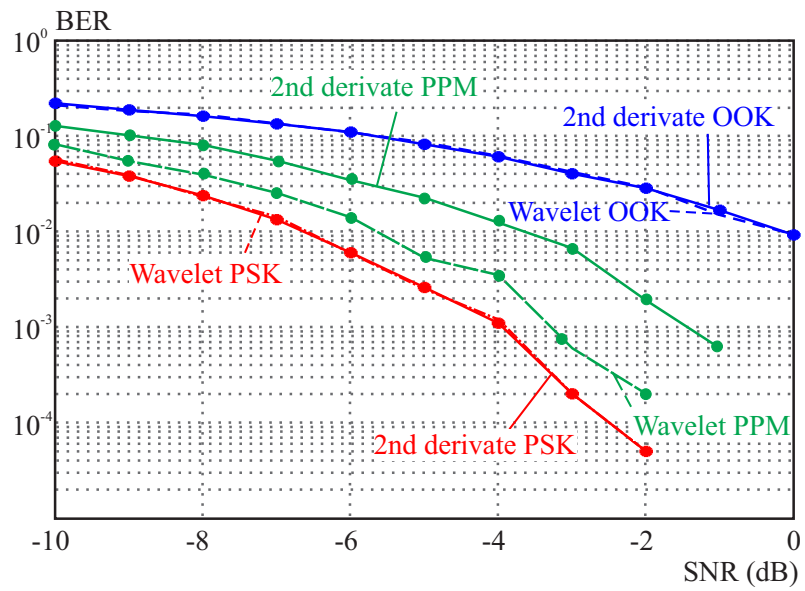

Fig. 8. BER performances in AWGN channel using wavelet based and Gaussian second derivative monopulse

Figure 6 shows that BPSK gives the best performance for wavelet based monopulse in the presence of AWGN channel. Thus AWGN channel is better for the transmission of BPSK modulated pulse. OOK has the highest energy level to achieve the same bit error rate over BPSK and PPM for Rayleigh channel. We can summarize that BPSK modulation in AWGN channel environment gives the best performance of all three modulation schemes.

Figure 7 shows the BER comparison for three PPM schemes; such as Binary PPM, 4-PPM and 8-PPM. 2PPM has greater inter-symbol distance than 4-PPM and 8-PPM. Thus 4-PPM and 8-PPM require more energy to achieve the same bit error rate than 2-PPM. Moreover, the performance of UWB transmission in AWGN channel is better than Rayleigh fading channel for PPM modulation schemes.

Figure 8 shows the BER performances of wavelet based monopulse and Gaussian second derivative monopulse in AWGN channel for three modulation schemes. It can be noticed from the figure that the performances are same for PSK and OOK which means pulse shape does not affect the performance in PSK and OOK. For PPM wavelet based monopulse gives the better performance than Gaussian second derivative monopulse.

Figure 9 shows the BER performances of three monopulses - Gaussian first derivative, Gaussian second derivative and wavelet-based monopulse using PPM modulation. As AWGN channel gives the better performance than Rayleigh channel, thus we considered AWGN channel for this analysis. The simulation result shows that the wavelet based monopulse gives the best performance among the three monopulses.

\section{CONCLUSION}

In this paper, we have analyzed and compared the performances of two different UWB signal such as wavelet based mono-pulse and Gaussian second derivative monopulse. In AWGN channel these two wave-shapes have the same performances for PSK and OOK modulations,

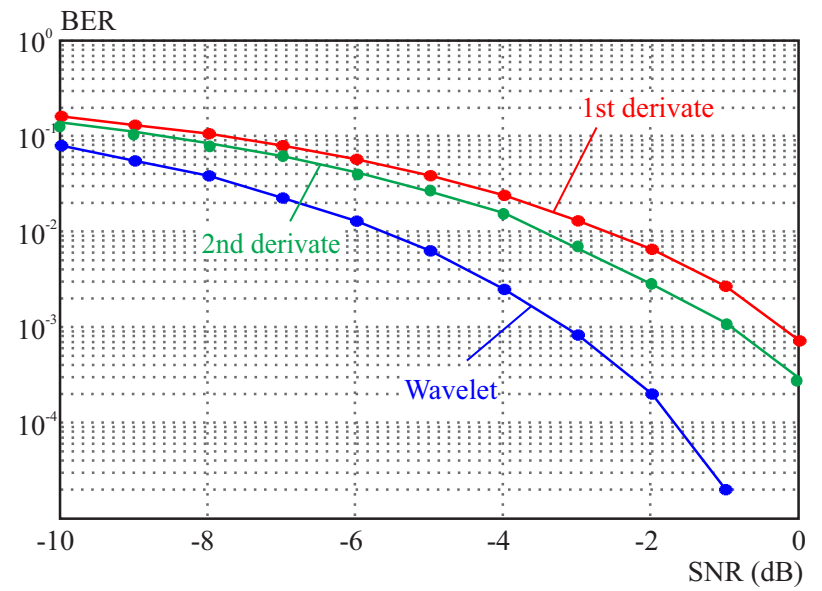

Fig. 9. BER performances for PPM modulation scheme for three monopulses

but wavelet based monopulse has the better performance for PPM modulation. Again, comparing the two pulse shapes, we get that wavelet-based monopulse gives the best BER performance. Moreover, we examined the BER performance of wavelet based monopulse for M-ary PPM schemes and the simulation results prove that the lower order modulation scheme gives better performance than the higher order schemes.

\section{REFERENCES}

[1] LIANG, Z. Zhou,Z.- : The Performance of UWB System - Influence of Monocycle Shape and Synchronization Timing Error, ECTI Transactions on Electrical Eng., Electronics, and Communications 3 No. 1 (Feb 2005).

[2] ZAMAN, A. A.-ISLAM, N.: Modulation Schemes and Pulse Shapping in Ultra-Wideband, IEEE, Southeastcon, 2008, pp. $142-146$.

[3] LEI, J.-JIANHUA, Z.-RUOJU, L.-PING, Z.: A Novel Modulation for UWB based WSN, in Proc. Int. Conf. on Commun. Technol., Guilin, China, 27-30 Nov 2006.

[4] CHEN, X.-KIAEI, S. : Monocycle Shapes for Ultra Wideband System, in Proceeding of IEEE International Symposium on Circuits and Systems, vol. 1, Scottsdale, AZ, USA, May 2002, pp. 597-600.

[5] HUANG, X.-LI, Y.: Generating Near-White Ultra-Wideband Signals with Period Extended PN Sequences, In Proceedings of the IEEE Semiannual Vehicular Technology Conference, vol. 2, Rhodes, Greece, May 6-9, 2001, pp. 1184-1188.

[6] ZHAO, L.-HAIMOVIC, A. : Performance of Ultra Wideband Communications in the Presence of Interference, IEEE Journal on Selected Areas in Communications 20 No. 9 (Dec 2002), 1684-1691.

[7] FOERSTER, J.-GREEN, E.-SOMAYAZULU, S.-LEEPER, D.: Ultra-Wideband Technology for Short-or MediumRange Wireless Communications, Intel Technology Journal Q2 (2001).

[8] REED, J. H.: Introduction to Ultra-Wideband Communication System, Prentice Hall Publications.

[9] HAFEZ, M. A.-ALAGOZ, F.-HAMALAINEN, M.-AHO, M. L.: On UWB Capacity with respect to Different Pulse Waveforms, Second IFIP International Conference on Wireless and Optical Communications Networks, WOCN, IEEE, 2005.

[10] WIN, M. SCHOLTZ, R.: Ultra-Wide Bandwidth Time-Hopping Spread-Spectrum Impulse Radio for Wireless Multiple- 
Access Communications, IEEE Trans. on Comm. 48 No. 4 (Apr 2000), 679-691.

[11] SCHOLTZ, R. A.-WEAVER, R.-HOMIER, E.-LEE, J.HILMES, P.-TAHA, A.-WILSON, R.: Uwb Radio Deployment Challenges, in Proc. IEEE Int. Symp. Personal, Indoor and Mobile Radio Communication, vol. 1, London, U.K., Sep 2000, pp. $620-625$.

[12] SHAN, D. M.-CHEN, Z. N.-WU, X. H. : Signal Optimization for UWB Radio Systems, IEEE Transactions on Antenna and Propagation 53 No. 7 (July 2005).

[13] LI, Y.-HUANG, X. : The Spectral Evaluation and Comparison for Ultra-Wideband Signals with Different Modulation Schemes, in Proc. of SCI2000, the 2000 World Multiconference on Systemics, Cybernetics and Informatics, vol. VI, Orlando, Florida, USA, July 23-26 2000, pp. 277-282.

[14] RAMIREZ-MIRALES, F.-SCHOLTZ, R. A. : Multiple-Access with Time Hopping and Block Waveform PPM Modulation, 1998 International Conf. on Commun. (ICC '98), vol. 2, Los Angeles, CA, USA, June 7-11, 1998, pp. 775-779.

[15] D'SOUZA, M.-POSTUlA, A.: Novel Ultra-Wideband Pulse Spectrum Modulation Scheme, in Proc. 2003 IEEE Conf. on Ultra Wideband Systems and Technologies, Reston, VA, Nov 16-19, 2003, pp. 240-244.

[16] RAMIREZ-MIRALES, F.: Performance of Ultra-Wideband SSMA using Time-Hopping and M-ary PPM, IEEE J. on Selected Areas in Commun. 19 (June 2001), 1186-1196.

[17] YANG, L.-GIANNAKIS, G. B. : Optimal Pilot Waveform Assisted Modulation for Ultra-Wideband Communications, IEEE Trans. Wireless Commun. 3 No. 4 (July 2004), 1236-1249.

[18] MATIN, M. A.: Ultra Wideband Communications: Novel trends-System, Architecture and Implementation, InTech, Croatia, 2011.

Received 17 December 2011

Tasnuva Ali has completed her BSc in Electronics and Telecommunication Engineering from Daffodil International University, Dhaka in 2008. She is working as a Lecturer in the Department of Electronics and Telecommunication Engineering in Daffodil International University. Her current research interests include UWB communication, wireless communication, radio over fiber networks and NFC security issues.
Poppy Siddiqua has completed her BSc in Electronics and Telecommunication Engineering from Daffodil International University, Dhaka in 2007. She is working as a Lecturer in the Department of Electronics and Telecommunication Engineering in Daffodil International University. Her current research interests include UWB communication, wireless communication, radio over fiber networks and telemedicine systems.

Mohammad A. Matin is currently working at the department of Electrical and Electronic Engineering, Institut Teknologi Brunei (ITB), Brunei Darussalam as an Associate Professor. Before joining ITB, he was with the department of Electrical Engineering and Computer Science, North South University as an Associate Professor. He obtained his BSc degree in Electrical and Electronic Engineering from BUET (Bangladesh), MSc degree in digital communication from Loughborough University, $\mathrm{UK}$ and $\mathrm{PhD}$ degree in wireless communication from Newcastle University, UK. He has taught several courses in communications, electronics and signal processing at KUET, Khulna University, and BRAC University during his career. Dr. Matin was a visiting academic staff at the National University of Malaysia (UKM), University of Malaya (UM) etc/. He has published over 60 refereed journals and conference papers. He is the author of eight academic books and nine book chapters. He has presented invited talks in Bangladesh, and Malaysia and has served as a member of the program committee for more than 50 international conferences like, ICCVE 2013,ICC 2014, ICCSIT'09, IDCS'09, ICCSN'10, ICCSIT'10, ICCSN'11 etc. He also serves as a referee of a few renowned journals, keynote speaker and technical session chair of few international conferences like TENCON Spring 2013, MIC-CPE 2008, ICCIT 2008, ICMMT 2010, ICCIT 2010, IEEE GLOBECOM 2010 etc/. He is currently serving as a member of editorial board of several international journals such as IET Wireless Sensor Systems (IET-WSS), Journal of Electrical and Computer Engineering (JECE), Hindawi Publishing Corporation, IJCTE, etc/. and Guest Editor of special issue of IJDSN, IJCNIS etc. His current research interests include UWB communication, wireless sensor networks, cognitive radio, EM modeling, and antenna engineering.

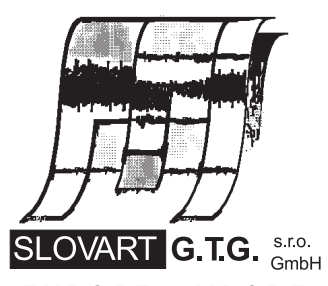

EXPORT - IMPORT
EXPORT - I M P OR T

of periodicals and of non-periodically printed matters, books and CD-ROMs

Krupinská 4 PO BOX 152, 85299 Bratislava 5, Slovakia tel: ++421 263839 472-3, fax: ++421263839485 info@slovart-gtg.sk; http://www.slovart-gtg.sk

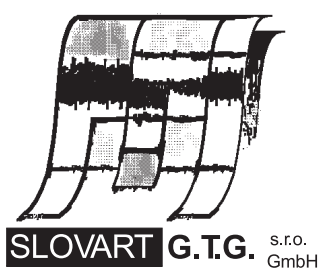

EXPORT - IMPORT 